\title{
Acupuncture and electro-acupuncture for people diagnosed with subacromial pain syndrome: a multicentre randomized trial
}

Jeremy Lewis ${ }^{1,2,3}$, Julius $\operatorname{Sim}^{4}$, Panos Barlas ${ }^{5}$

1 Department of Clinical Therapies, University of Limerick, Ireland

2 School of Health and Social Work, University of Hertfordshire, Hatfield, United Kingdom

3 Central London Community Healthcare NHS Trust, London, United Kingdom

4 Institute for Primary Care and Health Sciences, Keele University, Staffordshire, United Kingdom

5 School of Health and Rehabilitation, Keele University, Staffordshire, United Kingdom

\section{Correspondence:}

Jeremy Lewis; E-mail: jeremy.lewis@londonshoulderclinic.com

Accepted for publication in European Journal of Pain, 29 November 2016; doi:10.1002/ejp.1001 


\section{Abstract \\ Background:}

Musculoskeletal disorders have been identified globally as the second most common healthcare problem for 'years lived with disability', and of these shoulder conditions are amongst the most common, frequently associated with substantial pain and morbidity. Exercise and acupuncture are often provided as initial treatments for musculoskeletal shoulder conditions but their clinical effectiveness is uncertain. This study compared group exercise with group exercise plus either acupuncture or electro-acupuncture in patients with subacromial pain syndrome.

\section{Methods:}

Two hundred and twenty-seven participants were recruited to a three-arm parallel-group randomized clinical trial. The primary outcome measure was the Oxford Shoulder Score. Follow-up was post treatment, and at 6 and 12 months. Between-group differences (two comparisons: the exercise group versus each of the acupuncture groups) were analysed at 6 months. A similar comparison across all follow-up time points was also conducted. Data were analysed on intentionto-treat principles with imputation of missing values.

\section{Results:}

Treatment groups were similar at baseline, and all treatment groups demonstrated an improvement over time. Between-group estimates at 6 months were, however, small and nonsignificant, for both of the comparisons. The analyses across all follow-up time points yielded similar conclusions. There was a high rate of missing values ( $22 \%$ for the Oxford Shoulder Score). A sensitivity analysis using complete datagave similar conclusions to the analysis with missing valuesimputed.

\section{Conclusions:}

In the current investigation, neither acupuncture nor electro-acupuncture were found to be more beneficial than exercise alone in the treatment of subacromial pain syndrome. These findings may support clinicians with treatment planning.

\section{Significance:}

Shoulder pain is common and associated with substantial morbidity. Acupuncture is a popular treatment for shoulder pain. The findings suggest that acupuncture and electro-acupuncture offer no additional benefit over exercise in the treatment of shoulder pain of musculoskeletal origin. 


\section{Introduction}

Musculoskeletal disorders have been identified globally as the second most common condition associated with 'years lived with disability' (Vos et al., 2012). Of these disorders, shoulder conditions are amongst the most common (Charles et al., 2007). Pain is the most common symptom described by people experiencing musculoskeletal shoulder conditions and the most common reason for seeking treatment (van der Windt et al., 1995).

Deriving a definitive diagnosis for most musculoskeletal shoulder conditions is difficult. Orthopaedic tests have been developed to enable clinicians to establish the cause of shoulder symptoms (Magee, 2014). However, narrative and systematic reviews have challenged the usefulness of these tests as a means of assessing isolated individual structures (Lewis, 2009; Hegedus et al., 2012; Lewis et al., 2015).

For those suffering low back pain it is now acceptable to classify symptoms as non-specific, simple or mechanical back pain (Waddell, 2004), without a need to be structurally definitive, and for many clinical presentations, the same may be appropriate for the shoulder region (Lewis, 2009). Conditions such as traumatic shoulder dislocations, frozen shoulder, severe osteoarthritis, avascular necrosis and tumours are ones where diagnoses may be easier to establish than those involving the bursae, the range of rotator cuff pathologies and other tissues under and around the acromion. Accordingly, 'subacromial pain syndrome' may be a more appropriate clinical classification than a specific tissuebased diagnosis or unsubstantiated aetiological mechanism such as subacromial impingement syndrome (Lewis, 2011, 2015, 2016). Subacromial pain syndrome embraces: subacromial impingement (Haahr et al., 2005; Haahr and Andersen, 2006; Holmgren et al., 2012), partial-thickness (Kukkonen et al., 2014) and full-thickness (Kuhn et al., 2013) rotator cuff tears, as well as massive irreparable rotator cuff tears (Ainsworth, 2006). The main treatment for subacromial pain syndrome is exercise.

Although exercise is the main treatment for the majority of musculoskeletal shoulder conditions and has been demonstrated to be as effective as surgery (Haahr and Andersen, 2006; Ketola et al., 2013; Kukkonen et al., 2014), both exercise and surgery are frequently not fully curative and are often associated with recurrence and ongoing morbidity and pain (Linsell et al., 2006; Paloneva et al., 2013). Accordingly, many clinicians incorporate other treatments to reduce symptoms. Acupuncture and electro-acupuncture (acupuncture with electrical stimulation of the inserted needles) are other common methods of treating shoulder pain. Despite its popularity (Kleinhenz et al., 1999; Molsberger et al., 2010; Johansson et al., 2011), findings from research investigating acupuncture in the treatment of shoulder pain have proven to be equivocal (Berry et al., 1980; Roach et al., 1991; Kleinhenz et al., 1999; Guerra de Hoyos et al., 2004; Green et al., 2005; Johansson et al., 2005; Lathia et al., 2009; Molsberger et al., 2010), as well as controversial (e.g.

http://www.dcscience.net/?p=6089, www.which.co.uk/news/2014/01/five-surprising-facts-abouthealth-treatments-350088/).

Current research demonstrates uncertainty regarding the effectiveness of acupuncture in the management of musculoskeletal shoulder pain. Green et al. (2005) state that due to the small number of trials to date, and their clinical and methodological diversity, there is little to support or challenge the use of acupuncture for shoulder pain. Accordingly, more information is required regarding the effectiveness of acupuncture treatments in the treatment of shoulder conditions.

The aim of this study was to compare the benefits of group exercise, group exercise and acupuncture, and group exercise and electro-acupuncture in the treatment of people with musculoskeletal shoulder pain classified as subacromial pain syndrome.

Standards for Reporting Interventions in Controlled Trials of Acupuncture (STRICTA) were adhered to, and include:

(1) Acupuncture rationale;

(2) Details of needling;

(3) Treatment regimen;

(4) Other components of treatment; 
(5) Practitioner background; and

(6) Control or comparator interventions (MacPherson et al., 2010).

\section{Methods}

The design of the study was a prospective, multicentre, randomized clinical trial, with assessor blinding. Between 2007 and 2013, participants with shoulder pain were recruited from four United Kingdom National Health Service (NHS) physiotherapy clinics. Three of these clinics were based in secondary healthcare teaching hospitals and one was based in a primary healthcare community clinic. Participants were referred to the physiotherapy clinics by general practitioners, consultant physicians and surgeons, and other physiotherapists.

Potential participants presenting with shoulder pain were identified prior to commencing treatment and were given both oral and written information about the study. Those interested in learning more about the study were contacted by the principal research physiotherapist at each location. Subject to the study inclusion and exclusion criteria (Table 1), participants who agreed to participate gave written informed consent and then underwent a baseline assessment.

At each site, participants were then randomized into one of three groups:

Group I: Shoulder advice and weekly exercise group (six 50-55-min sessions).

Group II: Shoulder advice and weekly exercise group (six 50-55-min sessions) together with six treatments of acupuncture.

Group III: Shoulder advice and weekly exercise group (six 50-55-min sessions) together with six treatments of electro-acupuncture.

Randomization was in a 1:1:1 ratio according to a sequence produced by a random number generator; a separate sequence was generated for each study site. Sequentially numbered opaque envelopes ensured concealment of groupallocation.

\subsection{Ethics}

Participants were fully informed of the study, including their right to withdraw from the investigation at any stage without the need to explain their decision, and were told that subsequent care would not be compromised by this decision. Ethical approval was granted by the NHS National Research Ethics Committee (07/Q0401/2).

\subsection{Outcome measures}

The primary outcome measure for this investigation was the Oxford Shoulder Score (OSS; Dawson et al., 2009). This is a 0-48 health-related quality of life scale on which higher scores are better. Secondary outcome measures were: the Shoulder Pain and Disability Index (SPADI), a 0-100 pain and disability scale on which lower scores are better (Roy et al., 2009); night pain; analgesic use; impact of main functional problem (0-10 scale on which lower scores are better); and shoulder range of movement. Two orthopaedic tests (Neer sign, Hawkin's test) were also included, not as tests to implicate a condition or structure, but as procedures to elicit symptoms associated with subacromial pain syndrome. Outcome measures were obtained prior to randomization, at the end of treatment (6 weeks), at 6 months, and at final follow-up at 12 months. The 6-month data collection was the primary outcome time point. The data collected and the time points at which this occurred are detailed in Table 2. Shoulder flexion, abduction, external rotation and internal rotation ranges of movement were measured as described by Valentine and Lewis (2006).

\subsection{Participant, therapist and assessor blinding}

The nature of the interventions did not permit the therapists providing the intervention or the participants receiving the intervention to be unaware of group allocation. However, assessors who collected all the outcome data were blind to participants' group allocation at every data collection time point. 


\subsection{Procedures}

A generic shoulder advice and exercise class that might be classified as light to moderate level of exercise, attended by all participants, was conducted once a week for 6 weeks as a circuit program. The class included: (1) warm-up exercises (e.g. static exercise bike); (2) shoulder range of movement exercises; (3) resisted internal and external rotation exercises; (4) generalized shoulder strengthening exercises (e.g. pulling and pushing against resistance); (5) weight-bearing exercises (e.g. pushing Swiss ball against wall, weight through arms on Swiss ball); (6) lower limb exercises (e.g. step-ups with concomitant upper limb elevation); and (7) cool-down exercises. Participants who were randomized to receive acupuncture or electro-acupuncture received these additional treatments twice a week for the first 3 weeks of the 6 -week shoulder programme. At each site, the shoulder advice and exercise class was conducted by two qualified physiotherapists.

Three acupuncture protocols were used, comprising: (1) an anterolateral shoulder pain protocol; (2) a posterolateral shoulder pain protocol; and (3) a general shoulder pain protocol (Table 3). The acupuncture point protocols were designed to correspond to the most common clinical presentations of shoulder pain and to allow some flexibility for the practitioner to modify according to individual patients' complaints; each protocol comprised points with which the practitioners were very familiar. Each participant received the protocol most relevant for his or her pain presentation. The acupuncture treatments (number of points, duration of treatment and frequency of treatment) were based on recommendations for adequate intervention (White et al., 2008). Needles were inserted unilaterally, on the side of symptoms, and up to six local needles were used as well as two distal needles (forearm or lower leg). Each acupuncture treatment lasted $30 \mathrm{~min}$; the timing of each treatment started after the last needle was inserted.

The acupuncture needles used in this investigation were single-use Seirin $0.25940 \mathrm{~mm} \mathrm{~W} / \mathrm{T}$, Seirin $0.25950 \mathrm{~mm} \mathrm{~W} / \mathrm{T}$, Seirin $0.25940 \mathrm{~mm}$ metal and Seirin $0.25950 \mathrm{~mm}$ metal (supplied by Scarsboroughs Ltd, Somerset, UK).

In the acupuncture group the needles were stimulated manually and in the electro-acupuncture group the intensity of stimulation was controlled via the stimulator. Typically, the therapists inserted all points intramuscularly (provided there was a muscle under the point) and stimulated all points every 3-5 min in order to maintain 'Deqi' for the duration of the treatment.

In addition to receiving general information about the research, all participants were made aware of any potential risks and benefits of acupuncture and electro-acupuncture, and were provided with acupuncture information sheets, acupuncture health screening questions, and an acupuncture consent form. All participants went through this process as part of the pre-randomization assessment procedures to ensure that they were aware of the possible interventions and risks and that no participant would be excluded from these treatments on health grounds following randomization. As recommended by the Acupuncture Association of Chartered Physiotherapists (AACP; a special interest group of the UK Chartered Society of Physiotherapy), routine pre- and post-acupuncture treatment advice was provided to all participants.

All physiotherapists providing acupuncture and electro-acupuncture treatments had completed a minimum of $80 \mathrm{~h}$ training in acupuncture. This period of training is based upon the UK Department of Health recommendation for health professionals (doctors, physiotherapists) wishing to provide acupuncture in the NHS. This is also the requirement of the UK Chartered Society of Physiotherapy and the UK Health and Care Professions Council. These practitioners do not use the protected title 'acupuncturist' unless registered with the British Acupuncture Council. Although having expert musculoskeletal knowledge with respect to anatomy, pathology and physiology, the physiotherapists providing the acupuncture treatment were not required to make a Traditional Chinese Medicine diagnosis or apply specialist techniques such as moxa. All practitioners were very familiar with all the points used in the protocols. As a requirement of the AACP, all physiotherapists who provided the acupuncture treatment participated annually in continuing professional development training. 